\title{
Finite Characteristic Ratio Constitutive Modelling for Geo-Materials
}

\author{
Zhang-Ming $\mathrm{Li}^{*}$, Xi Wang and Xin-Li Hu \\ Inst. of Geotechnical Engineering, Guangdong University of Technology, \\ Guangzhou, China \\ ukzmli@163.com*
}

\begin{abstract}
The constitutive modeling relation of finite characteristic ratio theory (FCRT) for geomaterials is presented. The finite characteristic ratio $L$, the ratio of a material internal measurement which represents statistic mechanical properties of a geo-material to the material external measurement, is considered as a phenomenological variable concerned with irrecoverable process; furthermore, the FCRT constitutive model for dilatancy of sand is originally proposed, and the accurate determination method of the only four nonelastic material parameters in the model are presented based on the deformation feature of sand. The dilative shearing hardening response of sand is described correctly; especially, the relationship among the responses of sands with different particle size is initially formed upon the FCRT model. The comparing results between the FCRT model and tests shows the FCRT model can describes the different properties of sands which are of different grain sizes naturally and the depiction of the model is conformed well by the sand tests.
\end{abstract}

Keywords: Finite characteristic ratio theory (FCRT), Geo-materials, Constitutive model, Parameters determination, Sand test, Particle size effect

\section{Introduction}

It is well established from numerous laboratory tests and engineering practices that the mechanical responses of geo-materials mainly depend on the meso-scale (granular scale) mechanism for most soils [1-8], and even macro-scale mechanism for some rocks [9-12]; in other words, the characteristic ratio L of a material point (internal) characteristic measurement to the material or engineering structure's external measurement is not small enough. Then, the analysis of a geotechnical problem might not include enough material points (namely volumetric elements in continuum mechanics) to adequately represent the mechanical behaviour of the whole. It means that the consideration of the "mathematical point" on the volume element might not be correct in geotechnical engineering generally. Therefore, a new descriptive method or theory is required to be developed for consideration of the effects of inter and/or outer characteristic measurement for a geomaterial on the mechanical responses of the geo-material.

\section{Basic Constitutive Relations of Finite Characteristic Ratio Theory}

The Finite Characteristic Ratio Theory (FCRT) was originally presented based on the observations [1] that (1) the material point which represents statistic mechanical properties of a geo-material is a finite geometry point but is not a mathematical point, (2) the finite characteristic ratio $\mathrm{L}$ defined above is a basic phenomenological variable which is related to the irrecoverable deformation and energy dissipation, and (3) $\mathrm{L}$ can be expressed as a function of stress or strain, temperature, and internal variables. With the 
above concepts, the new constitutive relations are derived within the general frame of thermodynamics with internal variables.

For determining the mechanical effect of the particles in geometrical size and the number of particles in any object or material, the characteristic length ratio $\mathrm{L}$ is defined as:

$L=L_{p} / L_{s}$

where $L_{s}$ is the characteristic length for any object or material, termed the external characteristic measurement, related to the initial property of any object or material; $\mathrm{L}_{\mathrm{p}}$ represents the size of a particle group or volume element, which represents statistically the mechanical properties of the object at a time, termed the internal characteristic measurement. It is usually expressed as a function of stress $\sigma_{\mathrm{ij}}$ or strain $\varepsilon_{\mathrm{ij}}$, temperature $\mathrm{T}$ and internal variable q. Similarly $\mathrm{L}$ also depends on those quantities mentioned above, and is expressed by the following relation:

$L=f\left(\sigma_{i j}, T, q ; \varepsilon_{i j}, T, q\right)$

Usually it is assumed that free energy inside any object consists of two parts: reversible and irreversible deformation and they are:

$G=G^{r}+G^{i}$

where $\mathrm{G}$ is the Gibbs free energy; $r$ and $\mathrm{i}$ represent the reversible and irreversible components respectively; $\mathrm{G}^{\mathrm{r}}$ is the elastic energy and $\mathrm{G}^{\mathrm{i}}$ is the dissipative energy, which is a function of the characteristic length ratio L:

$G^{i}=G^{i}(L)$

where $\mathrm{L}$ is replaced by its initial value $\mathrm{L}_{0}$ and then $\mathrm{G}^{\mathrm{i}}$ is developed by Taylor's series:

$G^{i}=G^{i}\left(L_{0}\right)+G^{i^{\prime}}\left(L_{0}\right)\left(L-L_{0}\right)+(1 / 2) G^{i^{\prime \prime}}\left(L_{0}\right)\left(L-L_{0}\right)^{2}+\ldots \ldots$

Those terms which are higher than second order are neglected because L is small. So equation (5) is written as:

$G^{i}=G^{i}\left(L_{0}\right)+G^{i}\left(L_{0}\right)\left(L-L_{0}\right)+(1 / 2) G^{i^{\prime \prime}}\left(L_{0}\right)\left(L-L_{0}\right)^{2}$

The first term $\mathrm{G}^{\mathrm{i}}\left(\mathrm{L}_{0}\right)$ for equation (6), under an external condition represents the dissipation energy corresponding to the irreversible deformation process of an object. Without considering the preloading pressure history of a solid mass, $\mathrm{G}^{\mathrm{i}}\left(\mathrm{L}_{0}\right)$ is zero and the second order tensor will be neglected if $L$ is very small. Finally $\mathrm{G}^{\mathrm{i}}(\mathrm{L})$ can be expressed as:

$G^{i}=G^{i}\left(L_{0}\right)+G^{i^{\prime}}\left(L_{0}\right)\left(L-L_{0}\right)$

From reversible thermodynamics,

$\varepsilon_{i j}=-\partial G / \partial \sigma_{i j}$

Alternatively, it may be expressed at small strain level as:

$\varepsilon_{i j}=\varepsilon_{i j}^{r}+\varepsilon_{i j}^{i}$

where $\varepsilon^{\mathrm{r}}{ }_{\mathrm{ij}}$ is reversible strain and $\varepsilon_{\mathrm{ij}}^{\mathrm{i}}$ is irreversible strain. Combining equation (3) with (8) and (9), these two important items can be given as follows:

$\varepsilon_{i j}^{r}=-\partial G^{r} / \partial \sigma_{i j}$ 
$\varepsilon_{i j}^{i}=-\partial G^{i} / \partial \sigma_{i j}$

where $\mathrm{G}^{\mathrm{i}}$ is given by equations (5), (6) or (7). Equations (8), (9), (10) and (11), and (5), (6) or (7) are the basic constitutive relations of the finite characteristic ratio theory. The following evolution relation is derived for the condition of a homo-thermal process [9]:

$d q / d z=f\left(\sigma_{i j}\right)$

where $\mathrm{z}$ is the so-called intrinsic time, which is a function related to the irreversible deformation accumulation.

\section{A Fcrt Model for Geo-materials}

\subsection{A Fcrt Model}

The characteristic length ratio $\mathrm{L}$ for geo-materials has a close correlation with deviatoric stress, mean stress (or static water pressure) and internal variables. In order to evaluate the dilative shear failure and hardening property of the material, L in the consistent condition, will be given as follows if the temperature is constant:

$L=-L_{0}\left(a_{1} I_{1}^{2}+a_{2} J_{2}-a_{3} S_{i j} q_{i j}-a_{4} q_{i j} q i_{j}-1\right)$

where $I_{1}$ is the first stress invariant, $J_{2}$ the second deviatoric stress invariant, $S_{i j}$ the deviatoric stress, $q$ the internal variable of second order, which is usually expressed by the irreversible volume accumulation. $\mathrm{a}_{1}, \mathrm{a}_{2}, \mathrm{a}_{3}$ and $\mathrm{a}_{4}$ are positive constants for different and $\mathrm{L}_{0}$ is the initial value of L. Combining equation (7) with (11) and (13), yields a new relation for $\varepsilon_{\mathrm{ij}}^{\mathrm{i}}$ as follows:

$\varepsilon_{i j}^{i}=L_{G 1}\left(2 a_{1} \sigma_{k k} \delta_{i j}+a_{2} S_{i j}-a_{3} q_{i j}\right)$

where $\mathrm{L}_{\mathrm{Gl}}=\mathrm{G}^{\mathrm{i}}\left(\mathrm{L}_{0}\right) \mathrm{L}_{0}$ is the non-negative constant, which should be multiple of $\mathrm{L}_{0}$, $\sigma_{\mathrm{kk}}=\mathrm{I}_{1}$, and $\delta$ is Kronecker $\delta$.

Another relation is obtained by combining equation (12) with (14a):

$d \varepsilon_{i j}^{i}=L_{G 1}\left[\left(2 a_{1} d \sigma_{k k} \delta_{i j}+a_{2} d S_{i j}-a_{3} F\left(\sigma_{i j}\right) d z\right)\right]$

where $\mathrm{F}\left(\sigma_{\mathrm{ij}}\right)$ is a tensor function which is related to the deviatoric stress, and mean stress, in general. Considering a non-coupling relation of the stress' deviatoric quantity with the volumetric quantity, a new relation should be as follows:

$F\left(\sigma_{i j}\right)=F_{1}\left(S_{i j}\right)+F_{2}\left(\sigma_{k k}\right)$

Then the irreversible volumetric deformation can be expressed as:

$d \varepsilon_{k k}^{i}=L_{G 1}\left[2 a_{1} d \sigma_{k k}-a_{3} F\left(\sigma_{k k}\right) d z\right]$

or

$d \varepsilon_{k k}^{i}=L_{G 1}\left(2 a_{1} d \sigma_{k k}-a_{3} d q_{k k}\right)$

In total quantity form, we have:

$\varepsilon_{k k}^{i}=L_{G 1}\left(2 a_{1} \sigma_{k k}-a_{3} q_{k k}\right)$

As the internal variable is usually expressed in the irreversible volume accumulation, the deviatoric part of the strain is:

$d e_{i j}^{i}=L_{G 1} a_{2} d S_{i j}$

or 
$e_{i j}^{i}=L_{G 1} a_{2} S_{i j}$

\subsection{Discussion of Parameters in the Constitutive Model}

The results from normal compression and cyclic-loading tests have shown that both medium dense and dense saturated sand, in drained or un-drained conditions, will have the properties of shearing dilatancy and hardening at certain stress levels [8]. After soil samples change in phase and enter a dilatancy zone, the ratio of deviatoric stresses to confining pressure tends to be in a critical state (a failure plane in three-dimensional stress state), and remains a constant, which increases with deviatoric stresses q, the mean normal pressure p, as shown in Figure 1.

Dilative shear failure will occur with an increase of shearing strain, and which in practice could be more easily caused by a strong earthquake for example. It is imperative to set up a constitutive relation for describing this failure. The purpose of this paper is, with reference to the finite characteristic ratio theory, to consider how to set up the appropriate constitutive relations and identify their natural meaning.
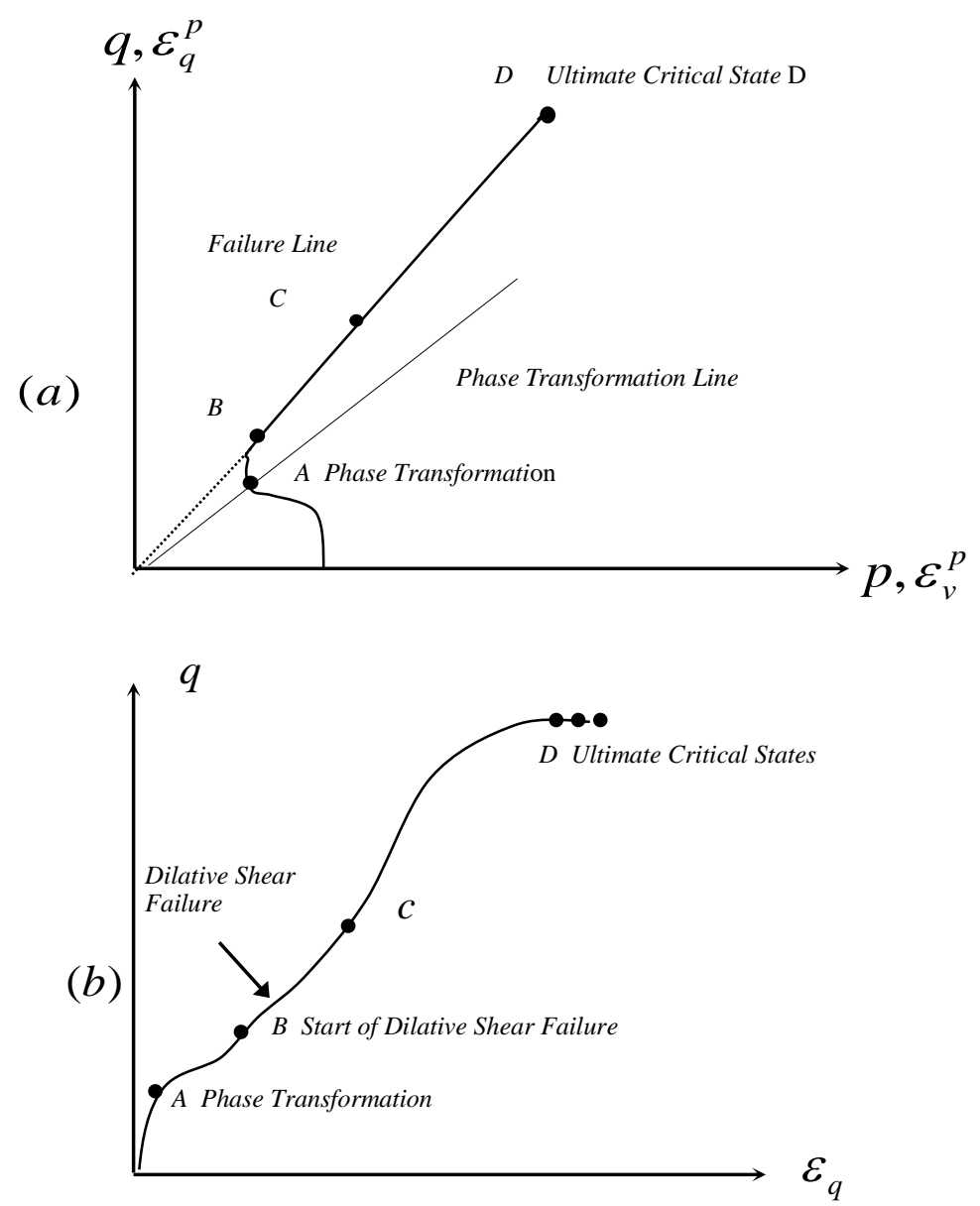

Figure 1. The Process of Dilative Shear Failure of a Soil Mass

In order to explain the natural meaning of the parameters in the constitutive relations mentioned earlier, triaxial tests for soil sample in un-drained condition will be discussed. As it is well known that compressive stress or strain are positive from the accustom expression of geomechanics.

In the axi-symmetric stress condition, combining equation (16) with (17), it can be solved through a few mathematic steps: 
$d q_{k k} /(d q D)=2 a_{2} /\left(3 a_{3}\right)-6 a_{1} /\left(a_{3} M D\right)$

$D=d \varepsilon_{k k}^{i} / d \varepsilon_{q}^{i}$

$q=\sigma_{11}-\sigma_{33} \quad p=\sigma_{k k} / 3$

$M=q / p$

$\varepsilon_{q}=2\left(\varepsilon_{11}-\varepsilon_{33}\right) / 3$

And the rest are the same as those relations mentioned above (note: $\mathrm{dq}_{\mathrm{kk}}$ is still the increment of internal variable. $\mathrm{dq}$ is the increment of stress difference $\mathrm{q}$, as expressed in Figure1).

In the following, the property of dilative shear failure will be employed to determine the parameters of the constitutive model mentioned above. Figure 1 has shown that there is a criterion of dilative shear failure in un-drained condition:

$q-M p=0, d \varepsilon_{k k}=0, d p>0$

From the elastic constitutive relations, we will find:

$d \varepsilon_{k k}^{r}=d p / K, d \varepsilon_{q}^{r}=d q /(3 G)$

where $\mathrm{K}$ and $\mathrm{G}$ are elastic volume modulus and shear modulus respectively and another form for equation (20) is:

$d \varepsilon_{k k}=d \varepsilon_{k k}^{r}+d \varepsilon_{k k}^{i}, d \varepsilon_{q}=d \varepsilon_{q}^{r}+d \varepsilon_{q}^{i}$

Combining equation (19) with (20) and (21), another relation is obtained:

$d \varepsilon_{q} / d q=1 /(3 G)-1 /(K M D)$

Comparing equation (18) with (22), we find:

$K=a_{3} /\left(6 a_{1}\right)$

$G=a_{3} /\left(2 a_{2}\right)$

$d \varepsilon_{q}=d q_{k k} / D$

where $\mathrm{a}_{1}$ is the parameter corresponding to response of materials under mean pressure, $\mathrm{a}_{2}$ is a coefficient related to the material's shape change, and $a_{3}$ change of inner structure.

Equation (16), (17) and the first two of equation (23) (4 equations in total) show that it is easy to obtain $S_{\mathrm{ij}}$ from normal tri-axial tests and not so hard to determine $\varepsilon^{\mathrm{i}}{ }_{\mathrm{kk}}$ and $\mathrm{e}_{\mathrm{ij}}^{\mathrm{i}}$ from cyclic-loading tests, and $\mathrm{q}_{\mathrm{kk}}$ is determined through the last formula of the equation (23) or (12). The elastic bulk modulus $\mathrm{K}$ and the shearing modulus $\mathrm{G}$ are obtained from the equal pressure test in three directions and pure shear test. Finally there are only four constants $a_{1}, a_{2}, a_{3}$ and $a_{4}$ to be determined in these four dependent equations. So these four constants can be completely given by the four equations mentioned. So far, all of the parameters required for determining the dilative shear failure can be determined.

From the viewpoint of the process of determining of the parameters of the constitutive model mentioned, there is clear natural meaning for each parameter, which can prevent them from being determined arbitrarily.

Under the tri-axial condition, when $i=1, j=1$, equation（17a) becomes:

$d \varepsilon_{q}=\frac{2}{3} L_{G 1} a_{2} d q$

where $d \varepsilon_{q}$ is the deviatoric strain-increment, and $d q$ is a deviatoric stress-increment defined as in soil mechanics; $L_{G 1}$ is related to the grain (or inner) size of the soil particles, and also related to the external characteristic size, which is the ratio between the two values. According to the basic meaning, it is taken as:

$L_{G 1}=L_{G 1}\left(d_{50}, \Phi\right)=b_{1} \frac{d_{50}^{\frac{1}{4}}}{\Phi}$ 
where $b_{1}$ is a material parameter related to the characteristic ratio, $d_{50}$ is the average grain size of soil, $\Phi$ is the outer characteristic size and here is taken as the diameter of the triaxial sample.

In addition, if the second order of $\mathrm{L}$ is maintained, (in the case of large-sized particle and the soil sample unchanged in size), the irreversible strain $\varepsilon$ can be kept, and the irreversible strain $\varepsilon$ only consisting of first order of $L$ can be expressed as:

$\varepsilon_{i j}^{i L}=\left[1+\left(L_{G 1} / L_{G 2}\right)\left(L-L_{0}\right)\right] \varepsilon_{i j}^{i}$

where $L_{G 2}=G^{i "}\left(L_{0}\right) L_{0}$ is a material constant which is function of $\mathrm{L}_{0}$, non-negative.

Obviously, always $L>L_{0}$, then $\left[1+\left(L_{G 1} / L_{G 2}\right)\left(L-L_{0}\right)\right]>1$, so $\varepsilon_{i j}^{i L}>\varepsilon_{i j}^{i}$.

Equation (27) shows that, if the stress and classification of the sand are unchanged, the irreversible deformation for a large-sized soil mass is higher than that for a small-sized one. Thus it can predict the influence of the material's internal structure on its properties. It is thought to be one of the important features of finite characteristic ratio theory.

\section{Tri-Axial Tests and Determination of the Model Parameters}

\subsection{Compression}

The Auto-controlling KTG Triaxial Testing Equipment is strain controlled. Test types including undrained tests, consolidated-undrained tests, and consolidated-drained tests etc. could be selected by the computer controlling programme, and the shear strain rate could be adjusted depending upon the test type. The test data are stored within the hard disk of computer.

The dry density of the sands and the initial condition of the tests are shown in Table 1 . The test results are presented on Figure 2 and Figure 3.

Table 1. The Dry Density of the Sand Samples and the Initial Condition in Tests

\begin{tabular}{|c|c|c|c|c|c|c|c|}
\hline Sand type & $\begin{array}{c}\text { Dry density } \\
\left(\mathrm{g} / \mathrm{cm}^{3}\right)\end{array}$ & $\begin{array}{c}\text { Sample } \\
\text { name }\end{array}$ & $\begin{array}{c}\text { Moisture } \\
\text { Content } \\
(\%)\end{array}$ & $\begin{array}{c}\text { Initial } \\
\text { void } \\
\text { ratio } \\
\end{array}$ & $\begin{array}{l}\text { Initial confine } \\
\text { Pressure }(\mathrm{kPa})\end{array}$ & $\begin{array}{c}\text { Back } \\
\text { Pressure } \\
(\mathrm{kPa}) \\
\end{array}$ & \begin{tabular}{|c|} 
Degree of \\
saturation \\
$(\%)$
\end{tabular} \\
\hline \multirow{2}{*}{ Coarse } & \multirow{2}{*}{1.435} & $\operatorname{cs} 3$ & 41.6 & 0.598 & 200 & 280 & 99 \\
\hline & & cs5 & 40.9 & 0.587 & 200 & 280 & 99 \\
\hline \multirow{2}{*}{ Medium } & \multirow{2}{*}{1.434} & $\mathrm{zs} 1$ & 43.2 & 0.620 & 200 & 200 & 99 \\
\hline & & $\mathrm{zs} 2$ & 41.7 & 0.599 & 200 & 240 & 99 \\
\hline \multirow{2}{*}{ Fine } & \multirow{2}{*}{1.325} & $\mathrm{xs} 2$ & 51.6 & 0.685 & 200 & 260 & 99 \\
\hline & & xs3 & 48.5 & 0.642 & 200 & 200 & 99 \\
\hline
\end{tabular}




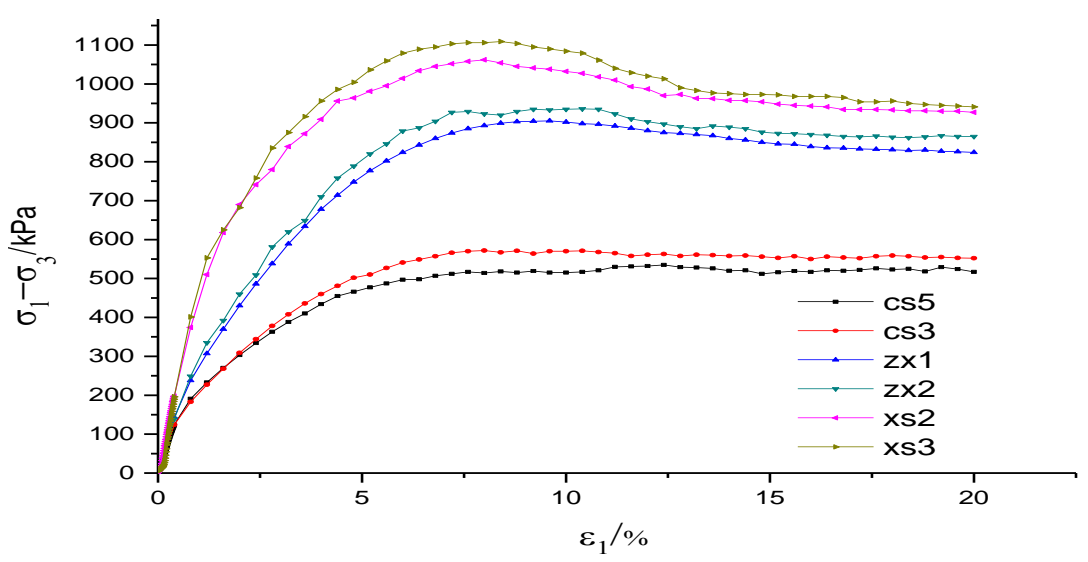

Figure 2. $\left(\sigma_{1}-\sigma_{3}\right)-\varepsilon_{1}$ Relations for the Coarse, Medium and Fine Sand from Triaxial Tests

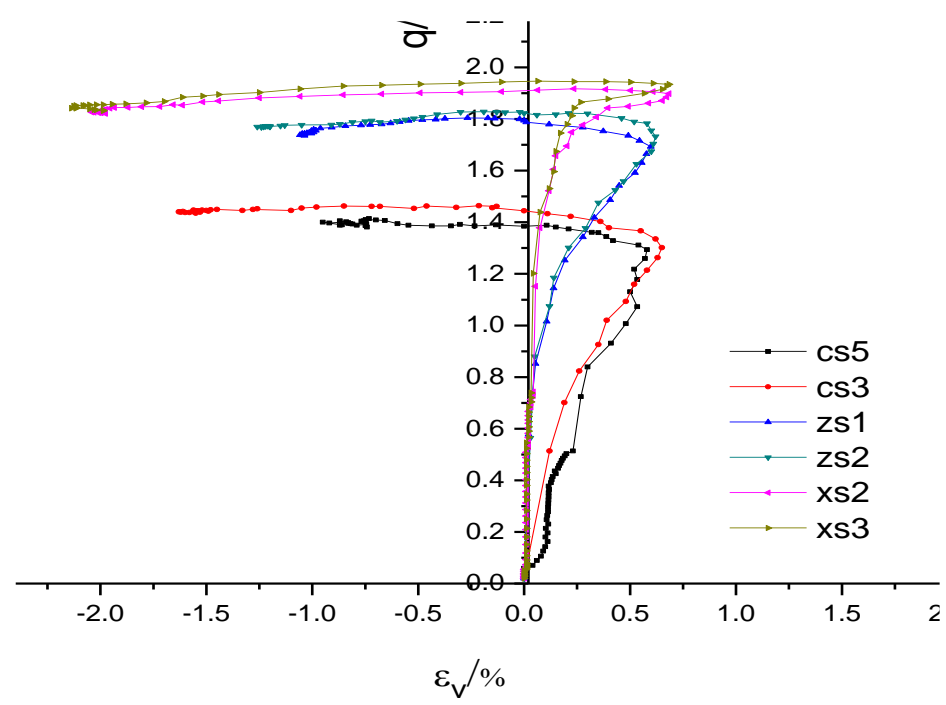

Figure 3-1. $\varepsilon_{v}-q / p$ Relations for the Coarse, Medium and Fine Sand from Triaxial Tests

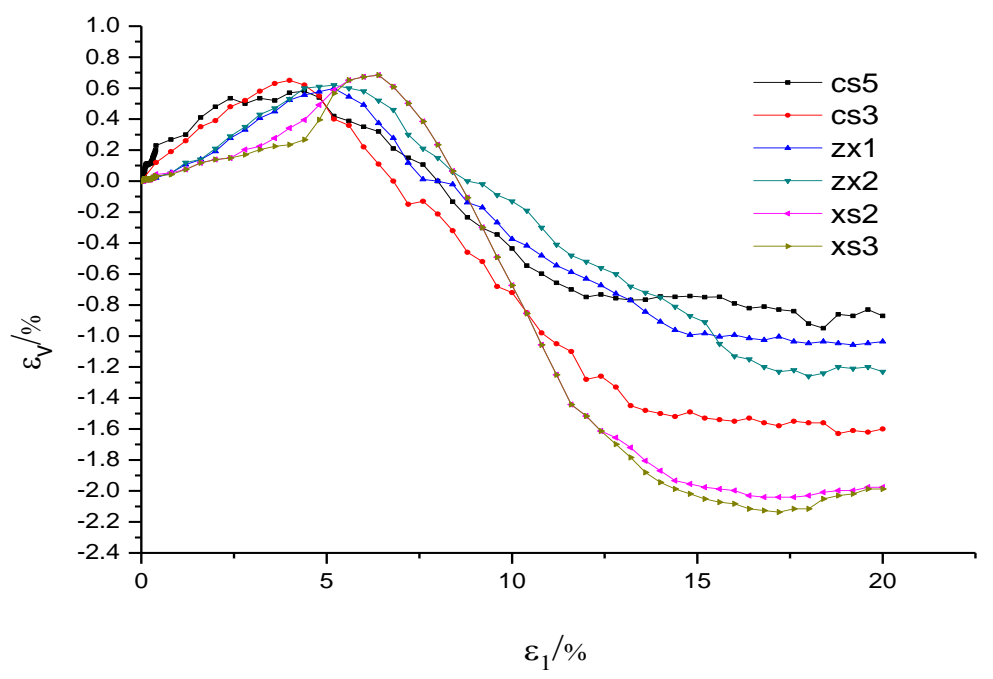

Figure 3-2. The $\varepsilon_{1}-\varepsilon_{v}$ Relations for the Coarse, Medium and Fine Sand from Triaxial Tests 


\subsection{Determination of Model Parameters}

\subsubsection{Calculation Relation under Tri-axial Pressure Condition}

Since the same constitutive model is adopted for the coarse, medium and fine sand the analysis is shown for the medium sand sample (No. zs1) as an example. The analyses for the other sands are carried out similarly same as that.

Under the tri-axial condition, since , $\sigma_{22}=\sigma_{33}=$ const, $\sigma_{11}=$ is variable, then, the total axial strain $\varepsilon_{11}$ is expressed by the recoverable and irrecoverable part $\varepsilon_{11}^{r}, \varepsilon_{11}^{i}$, $d \varepsilon_{11}=d \varepsilon_{11}^{r}+d \varepsilon_{11}^{i}$

The recoverable part can be expressed by linear elastic relation as

$d \varepsilon_{11}^{r}=\frac{1}{E}\left(d \sigma_{11}-2 v d \sigma_{33}\right)$

Under constant confining pressure, i.e.

$d \varepsilon_{11}^{r}=\frac{d \sigma_{11}}{E}$

The irrecoverable part under a constant confining pressure $\left(d \sigma_{33}=0\right)$ is expressed according to formula (14) as

$d \varepsilon_{11}^{i}=L_{G 1}\left[\left(2 a_{1}+\frac{2}{3} a_{2}\right) d \sigma_{11}-a_{3} d q_{k k}\right]$

\subsubsection{Determination of the Model Parameters}

The target function is taken as for determination of the parameters:

$F(X)=\left(\sum_{i=1}^{m}\left(\delta_{i}(X)-\delta_{i}^{*}\right)^{2}\right)^{\frac{1}{2}}$

where $\delta_{i}(X)$ is the calculation value, $\delta_{i}^{*}$ is a test value, and $\mathrm{X}$ is a reverse-analysis parameter. The target function should be minimized for seeking for $\mathrm{X}$ under a certain condition, i.e.

\section{(1) Determination of the parameter $a_{2}$}

From formula (24), we have

$a_{2}=\frac{3}{2 L_{G 1}} \frac{d \varepsilon_{q}}{d q}$

Due to $d \varepsilon_{q}$ can be expressed by $d q$, i.e. $d \varepsilon_{q}=\psi(d q), a_{2}$ could be expressed as the function of $d q$, i.e.

$a_{2}=\phi(d q)$

Upon the test data analyses, the expression of $a_{2}$ is obtained as

$a_{2}=\left(a_{0}+b_{0} q+c_{0} q^{2}\right) / L_{G 1}$ when $q \leq q_{0}$

$a_{2}=\left(a_{0}+b_{0} q+c_{0} q^{2}\right)^{-1} / L_{G 1}$ when $q>q_{0}$

The values of parameters $\mathrm{q}_{0}, \mathrm{a}_{0}, \mathrm{~b}_{0}$ and $\mathrm{c}_{0}$ for the coarse, medium and fine sand are shown in Table 2. 
Table 2. Values of Parameter $a_{2}$ for the Coarse, Medium and Fine Sands

\begin{tabular}{|c|c|c|c|c|c|}
\hline Sands & $\mathrm{q}_{0}(\mathrm{kPa})$ & Relation & $\mathrm{a}_{0}$ & $\mathrm{~b}_{0}$ & $\mathrm{c}_{0}$ \\
\hline \multirow{2}{*}{ coarse } & \multirow{2}{*}{502} & $\mathrm{q} \leq \mathrm{q} 0$ & $-4.2859 \mathrm{E}-5$ & $3.3887 \mathrm{E}-7$ & $-1.5486 \mathrm{E}-10$ \\
\cline { 3 - 6 } & & $\mathrm{q}>\mathrm{q} 0$ & 5743.9 & 70.328 & -0.12161 \\
\hline \multirow{2}{*}{ medium } & \multirow{2}{*}{748} & $\mathrm{q} \leq \mathrm{q} 0$ & $-1.1132 \mathrm{E}-5$ & $9.8955 \mathrm{E}-8$ & $-1.9731 \mathrm{E}-11$ \\
\cline { 3 - 6 } & & $\mathrm{q}>\mathrm{q} 0$ & -64740 & 255.24 & -0.19212 \\
\hline \multirow{2}{*}{ fine } & \multirow{2}{*}{986} & $\mathrm{q} \leq \mathrm{q} 0$ & $9.4859 \mathrm{E}-6$ & $-4.4990 \mathrm{E}-8$ & $6.4878 \mathrm{E}-11$ \\
\cline { 3 - 6 } & & $\mathrm{q}>\mathrm{q} 0$ & -3496.1 & 6817.9 & -3.2945 \\
\hline
\end{tabular}

(2) Determination of the evolution function $d q_{k k}$

From equations (12) and (15), we have

$d q_{k k}=F\left(\sigma_{k k}\right) \cdot d z$

The above formula can be also expressed as follows:

$d q_{k k}=H\left(d \varepsilon_{k k}, q\right)$

Based on test results we get

$d q_{k k}=a \cdot \operatorname{EXP}(b \cdot q)\left(c+d \cdot d \varepsilon_{k k}^{i}+e \cdot\left(d \varepsilon_{k k}^{i}\right)^{2}\right)$

The values of parameters $\mathrm{q}_{0}, \mathrm{a}, \mathrm{b}, \mathrm{c}, \mathrm{d}$ and e are shown in Table 3 .

Table 3. The Parameter Values of Evolution Function $d q_{k k}$ for the Coarse, Medium and Fine Sands

\begin{tabular}{|c|c|c|c|c|c|c|c|}
\hline Sands & $\mathrm{q}_{0}(\mathrm{kPa})$ & Relation & $\mathrm{a}$ & $\mathrm{b}$ & $\mathrm{c}$ & $\mathrm{d}$ & $\mathrm{e}$ \\
\hline \multirow{2}{*}{ coarse } & \multirow{2}{*}{502} & $\mathrm{q} \leq \mathrm{q}_{0}$ & 0.04202 & 0.00646 & \multirow{2}{*}{0.00298} & \multirow{2}{*}{-0.1389} & \multirow{2}{*}{-7.1428} \\
\hline & & $q>q_{0}$ & 1 & 0 & & & \\
\hline \multirow{2}{*}{ medium } & \multirow{2}{*}{748} & $\mathrm{q} \leq \mathrm{q}_{0}$ & 0.23067 & 0.00179 & \multirow{2}{*}{0.00557} & \multirow{2}{*}{-0.1470} & \multirow{2}{*}{-16.7340} \\
\hline & & $q>q_{0}$ & 1 & 0 & & & \\
\hline fine & - & - & 1 & 0 & 0.00713 & -0.1125 & -6.4335 \\
\hline
\end{tabular}

\subsection{Calculation and Analysis}

For the coarse sand, $d_{50}=1.5 \mathrm{~mm}$ (take the average values of $1 \sim 2 \mathrm{~mm}$ ), $\Phi=39.1 \mathrm{~mm}$, and Young's modulus E=32MPa, Poisson's ratio $v=0.42$ were got from the test.

To combine formula (23) with the relations of elastic constants in elasticity, we have

$K=\frac{E}{3(1-2 v)}=\frac{a_{3}}{6 a_{1}}$

$G=\frac{E}{2(1+v)}=\frac{a_{3}}{2 a_{2}}$

From formula (25)

$L_{G 1}=2.830 \times 10^{-2} b_{1}$

Then, based on (23b)

$a_{3}=2 a_{2} \times \frac{E}{2(1+v)}=2 \times a_{2} \times \frac{32 \times 10^{3}}{2(1+0.42)}=2.2536 \times 10^{4} \cdot a_{2}$

Combining equation (23a) with (38):

$a_{1}=\frac{a_{3}}{6} \times \frac{3(1-2 v)}{E}=\frac{1-2 v}{2(1+v)} a_{2}=\frac{4}{71} a_{2}$ 
The following demonstrates the calculation process. For the initial confining pressure $\sigma_{11}=\sigma_{22}=\sigma_{33}=200 \mathrm{kPa}$, the first level loading is carried out:

$q^{(1)}=141 \mathrm{kPa}, d \sigma_{11}^{(1)}=141 \mathrm{kPa}$,

then,

$p^{(1)}=\frac{\sigma_{11}+2 \sigma_{33}}{3}=247 \mathrm{kPa}$

and from the test $d \varepsilon_{k k}^{(1)}=d \varepsilon_{v}^{(1)}=0.13 \times 10^{-2}$, therefore, from equation (34a), $a_{2}=6.51 \times 10^{-5} / b_{1}$

upon (36a) , have $d q_{k k}=0.000292$

therefore,

$d \varepsilon_{11}^{i(1)}=L_{G 1}\left[\left(2 a_{1}+\frac{2}{3} a_{2}\right) d \sigma_{11}-a_{3} d q_{k k}\right]=0.0190 \times 10^{-2}$

The elastic strain is

$d \varepsilon_{11}^{r(1)}=\frac{d \sigma_{11}^{(1)}}{E}=0.441 \times 10^{-2}$

Total strain is $\varepsilon_{11}^{(1)}=d \varepsilon_{11}^{r(1)}+d \varepsilon_{11}^{i(1)}=0.460 \times 10^{-2}$

Other calculation is as above. The type calculation results on axial stress-strain of the coarse, medium and fine sands are shown in Table 4.

Table 4. The Model Calculation Results of Axial Stress-strain for the Coarse, Medium and Fine Sands

\begin{tabular}{|c|c|c|c|c|c|c|}
\hline \multirow{2}{*}{$\begin{array}{c}\text { Coarse } \\
\text { sand }\end{array}$} & $\sigma_{11}(\mathrm{kPa})$ & 341 & 509 & 660 & 710 & 770 \\
\cline { 2 - 7 } Medium & $\varepsilon_{11}(\%)$ & 0.460 & 2.023 & 4.050 & 5.055 & 7.689 \\
\cline { 2 - 7 } sand & $\sigma_{11}(\mathrm{kPa})$ & 343 & 630 & 914 & 1060 & 1104 \\
\hline \multirow{2}{*}{$\begin{array}{c}\text { Fine } \\
\text { sand }\end{array}$} & $\varepsilon_{11}(\%)$ & 0.416 & 1.974 & 4.392 & 6.859 & 8.998 \\
\cline { 2 - 7 } & $\sigma_{11}(\mathrm{kPa})$ & 453 & 862 & 1156 & 1279 & 1295 \\
\hline
\end{tabular}

\section{Conclusions}

Comparing the results of the model theory with the data of the tests as show in figure 4, it can be obtained as following:

(1) The axial stress-strain relation of FCRT theory model and that of tri-axial tests for the coarse, medium and fine sands match well. That shows the specialty of the FCRT model that the model can describe the different properties of sands which are of different grain sizes naturally; this response differences come from the difference of the (inner and outer) characteristic ratio.

(2) The FCRT model does not adopt the conception of "yield" which is an imagination for geo-materials; and this model can describe the initial non-linear and the coupling between recoverable and irrecoverable deformation easily.

(3) The parameter determination method of the model is quite easy; the parameters for given sand are determinate and the arbitrariness in the parameters determination is avoided. 


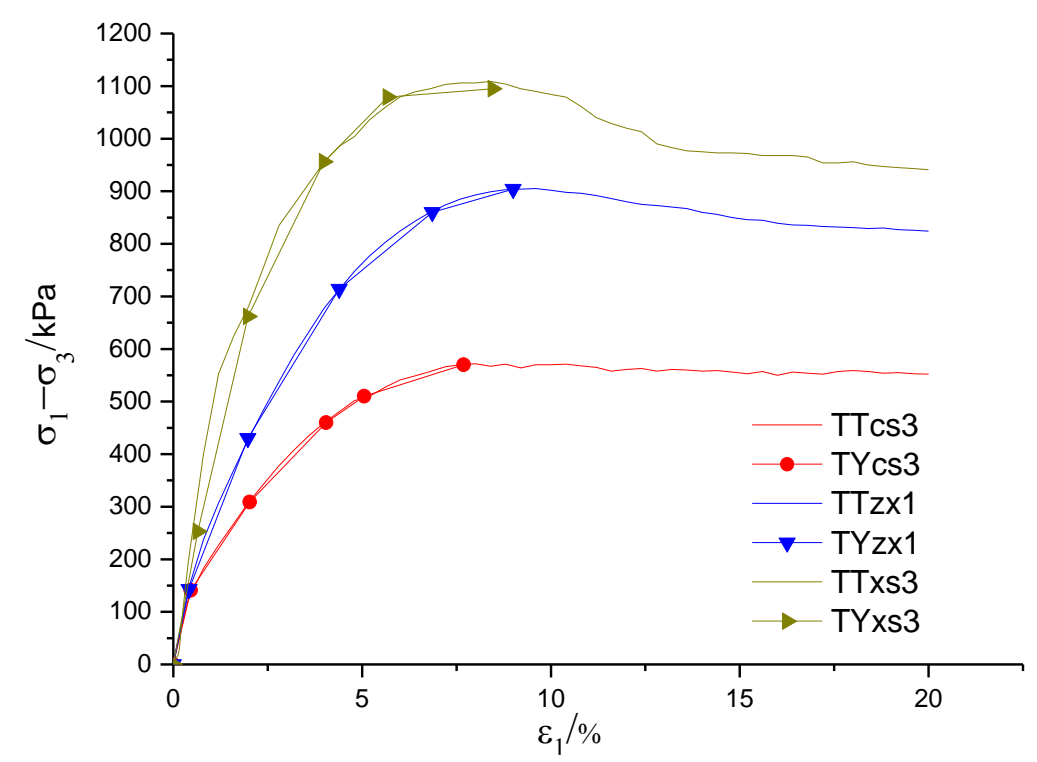

Figure 4. The Compare of Axial Stress-strain Relation Curves of FCRT Theory Model and Tri-axial Tests for the Coarse, Medium and Fine Sands (TT and TY represent the test and theory result respectively)

\section{Acknowledgment}

The authors gratefully acknowledge financial support provided by National Natural Science Foundation of China (Project Approval No. 51178122) and Natural Science Foundation of Guangdong Province ( Project Approval No. 2016A030313692).

\section{References}

[1] Li, Z. M. "Theory of finite characteristic ratio and its physical background", Chinese Jour. of Rock Mechanics and Engineering. 2000, 19[3]: 326 329(in Chinese)

[2] Bishop, A. W. and Henkel D. J. "The measurement of soil properties in the triaxial test", London: Edward Arnold. 1978, 33 144

[3] Li, Z. M. "Theory and engineering technology of ground improvement", Beijin: Chinese Electric Power Press, 2014 (in Chinese)

[4] Paulo J. Venda Oliveira, Luís J. L. Lemos. “ Experimental study of isotropic and anisotropic constitutive models", Journal of Geotechnical and Geoenvironmental Engineering. 2014, 140: 06014008-1 7

[5] Sand, S C. M. and Chandler, H. W. "Simulating pressure-induced compaction by grain rearrangement", Geotechnique Letters, 2012, 2: 187 192

[6] W. T. Sołowski and S. W. Sloan. "Equivalent stress approach in creation of elastoplastic constitutive models for unsaturated soils", International Journal of Geomechanics. 2015, 15: 04014041-1 13

[7] Sachen A, Penumadu D. "Effect of shear deformation on microfabric of clay using XRD technique", geotechnical and Geological Engineering, 2008, 6:1573 1529

[8] Li, X. S. "Modelling of dilative shear failure", Jour. of Geotechnical Engineering. 1997, 123(7):609 616

[9] Li, Z. M., Wang, W. L., Feng Y.X. " Generalized endochronic constitutive equation and the viscoplastical model for rock". Proceeding of the International Conf. on Nonlinear Mechanics, (Shanghai, Oct. 1985), Chien Wei-zang(Ed), Beijing: Science Press, 574 577

[10] Sheng Qian, Yang Yanshuang, et a1. "Study of size effect of rock mass deformation modulus based on in-situ test”. Rock and Soil Mechanics, 2010，31(9): 2875 2881(in Chinese)

[11] Li Zhangming. "A new approach to rock failure: criterion of failure in plastical strain space". Engineering Fracture Mechanics, 1990, V35 (4/5): $739-742$

[12] Li Zhangming,Wu Feng. "Finite characteristic ratio theory model analysis for the thin rock pillar of a twin tunnel". Applied Mechanics and Materials, 2011, 71-78: 4985-4988 


\section{Authors}

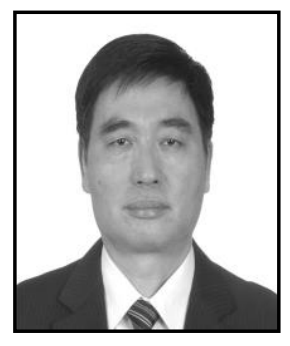

Zhang-Ming Li, professor in geotechnical engineering in Guangdong University of Technology, received his B.S. degrees in civil engineering from Shangdong University of Science and Technology in Jan.1982, M.S. degrees in geotechnical engineering from Chinese Academy of Sciences in Dec. 1984, and $\mathrm{Ph} . \mathrm{D}$. degrees in civil engineering from Huazhong University of Science and Technology in 2004. He has published over 120 papers in academic journals and international conferences, 8 books in publishing house, and 3 national and local regulations/codes. He has 5 China national invention patent, 4 software copyright, 3 high level science and technology award, and more than 40 leading the research projects; presided over complex foundation works more than 30 . He first proposed the Finite Characteristic Ratio Constitutive theory (FCRT), the static and dynamic drainage consolidation method for ground treatment, and successfully developed a high-energy impact test system and high-energy dynamic plate load tester. His research interests include theory and application of geotechnical engineering.

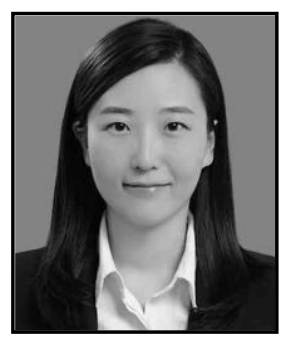

Xi Wang received her B.E. degrees in civil engineering from Zhengzhou University of Aeronautics in 2012. She is pursuing M.E. degrees in architectural and civil engineering at Guangdong University of Technology. Under the guidance of Professor Zhang-Ming Li, she has already published three journal papers. The topics of her current research interests include static-dynamic drainage consolidation method and evaluation of reinforcement effect.

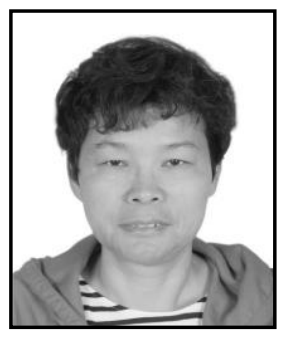

Xin-Li Hu, associate professor in civil engineering in Guangdong University of Technology, received her B.E. degrees in industrial and civil construction engineering and her Ph.D. degrees in structural engineering from Hunan University. She has already published more than twenty journal papers. 